\title{
Evaluación Del Efecto Aglutinante Del Almidón De Canna Indica L. (Achira), En La Elaboración De la Salchicha
} Vienesa

\section{Evaluation of the binding effect of Canna starch Indica L. (Achira), in the}

\author{
preparation of the Viennese sausage
}

Fabricio Guzmán Acán. ${ }^{1}$, Veronica de Angeles Bonifaz. ${ }^{2}$ \& Gabriela Vayas Castillo. ${ }^{3}$

\begin{abstract}
:
DOI: https://doi.org/10.33262/cienciadigital.v3i2.6.573

In the center of meat production at the ESPOCH, it was evaluated different starch of achira levels as binder $(5,10,15 \%)$, in the Viennese sausage type development , with three replications per treatment and a experimental $2 \mathrm{~kg}$ unit size and the obtained results were analyzed under a completely randomized design. It determined that the nutritional quality was not affected by statistically effect by the starch of achira addition for all treatments and presents average values of $69.68 \%$ of moisture, $13.66 \%$ protein, $11.12 \%$ fat and $3.09 \%$ ash. In the physical properties the Viennese sausage as the $\mathrm{pH}$ and the acidity provided no present a statistically significant difference between the averages reported for all treatments whose values were on the 5 th day $\mathrm{pH} 6.24$; 10 days 6.18 ; to the 15 days 6.04 and acidity to the 5 days $6.31^{\circ} \mathrm{D}$; to the 10 days $6.14^{\circ} \mathrm{D}$ and the 15 days $5.99^{\circ} \mathrm{D}$, both $\mathrm{pH}$ and acidity fluctuated in values considered acid, staying in the allowable range for this type of product, required in the INEN 1338 norm.
\end{abstract}

Keywords: Viennese sausage, Achira, Starch, Binder, Canna indica L.

\footnotetext{
1 Escuela Superior Politecnica de Chimborazo, Chimborazo, Ecuador, fabricio.guzman@espoch.edu.ec

2 Escuela Superior Politecnica de Chimborazo, Chimborazo, Ecuador, veronicabonifaz@g mail.com

3 Escuela Superior Politecnica de Chimborazo, Chimborazo, Ecuador, vayascastillo@gmail.com
} 


\section{Resúmen:}

En el Centro de Producción de Cárnicos de la ESPOCH, se evaluó diferentes niveles de almidón de achira como aglutinante $(5,10,15 \%)$, en la elaboración de salchicha vienesa, con tres repeticiones por tratamiento y un tamaño de la unidad experimental de $2 \mathrm{~kg}$, los resultados obtenidos se analizaron bajo un diseño completamente al azar. Determinándose que la calidad nutritiva no se vio afectada estadísticamente por efecto de la adición del almidón de achira se presentan valores promedio de $69.68 \%$ de humedad, $13.66 \%$ de proteína, $11.12 \%$ de grasa y $3.09 \%$ de cenizas. En las propiedades físicas como el $\mathrm{pH}$ y la acidez no presentó una diferencia estadística significativa entre las medias reportadas para todos los tratamientos cuyos valores fueron a los 5 días $\mathrm{pH}$ 6.24; a los 10 días 6.18; a los 15 días 6.04 y la Acidez a los 5 días $6.31^{\circ} \mathrm{D}$; a los 10 días $6.14^{\circ} \mathrm{D}$ y a los 15 días $5.99^{\circ} \mathrm{D}, \quad$ tanto el $\mathrm{pH}$ como la acidez fluctuaron en valores considerados ácidos, manteniéndose en el rango permisible para este tipo de producto, exigidos en la INEN 1338.

Palabras claves: Salchicha Vienesa, Achira, Almidón, Aglutinante, Canna indica L.

\section{Introduccion}

\section{Introduccion al problema}

En el Centro de Procesamiento de productos cárnicos de la Facultad de Ciencias Pecuarias de la Escuela Superior Politécnica de Chimborazo, en la actualidad se encuentra produciendo diferentes productos cárnicos, frescos, de pasta fina, gruesa, productos escaldados, ahumados y madurados. Siendo la salchicha tipo Vienesa, el producto estrella y de mayor aceptación por los consumidores. Se ha observado que este producto pese al buen proceso de elaboración y la aplicación de buenas prácticas de manufactura, e higiene, luego de la elaboración y conservación por un tiempo de 5 días, empieza un proceso de exudación de líquidos, que a pesar de ser mínimo es suficiente para ser perceptible por los consumidores.

El problema se presenta puesto que se utiliza materias primas de buena calidad nutritiva, pero no de buena calidad tecnológica, debido a que la carne de bovino proviene de animales criados básicamente con el propósito de producción de leche, que luego de terminar su vida productiva en ese campo, son faenados y enviados para la elaboración de embutidos.

Por Tal razón la carne utilizada de estos animales no tienen buenas características tecnologías, por ejemplo, la CRA (Capacidad de retención de agua), es muy débil, así como la CE (Capacidad de emulsión), baja considerablemente. Y por este motivo no se puede lograr productos de buena calidad sin la ayuda de sustancias que se puedan añadir a la emulsión del embutido para que evite 
una ruptura y separación de la fase sólida y liquida del embutido. Al observar este problema que se presenta en la elaboración de la salchicha tipo vienesa, se plantea reducir este defecto con la utilización del almidón de achira como agente aglutinante, Bonete, M. (2012) menciona que la achira posee una alta capacidad de retención de agua \% CRA 162.8\% para el sagú.

Debido a la constitución estructural de amilo pectina y amilasa, constituyentes que tienen la característica de aglutinar o ligar agua, en un product. Con la adición del almidón de achira se pretende mejorar la capacidad de retención de agua de la proteína de la carne de bovino y de esta manera evitar el problema de ruptura de la emulsión y asegurar una buena apariencia de la salchicha vienesa durante su periodo de almacenamiento, comercialización y consumo, sin afectar la calidad nutritiva del embutido.

Se determinó el nivel más adecuado de los tres porcentajes en estudio del almidón de Canna indica L (Achira), 5, 10, 15\%, que se adiciono en la formulación para la elaboración de la salchicha tipo vienesa, con la finalidad de evitar la ruptura de la emulsión del producto.

Una vez realizado el embutido salchicha vienesa, se procede a realizar el muestreo y se toma las muestras homogéneas, que debidamente codificadas serán almacenados a una temperatura de $5{ }^{\circ} \mathrm{C}$, para su posterior análisis en el laboratorio certificado, que nos brindara seguridad en los resultados a presentarse, en los parámetros de estudio como son la composición bromatológica, los análisis físicos y de característica tecnológica (CRA), análisis de $\mathrm{pH}$ y acidez para determinar la vida de anaquel..

\section{Importancia del problema}

La investigación del efecto aglutinante del almidón de Canna indica L (achira), en la elaboración de salchicha tipo vienesa es importante para los productores de productos cárnicos y las diferentes planta de procesamiento de cárnicos de la Facultad de Ciencias Pecuarias de la escuela Superior Politécnica de Chimborazo. Los consumidores prefieren adquirir y consumir alimentos de excelente calidad nutricional pero también aquellos que llaman la atención a sus sentidos especialmente la vista pues si se da una buena impresión a este sentido se asegura la comercialización del producto. Para lo cual se ha planteado el objetivo general

Evaluar el efecto aglutinante del almidón de achira en la elaboración de la salchicha Tipo Vienesa. Además se plantearon los siguientes objetivos específicos que ayudaran al desarrollo sistemático del estudio. 
Determinar cuál de los tres niveles de almidón de achira (5, 10 y 15\%) adicionados en la elaboración de Salchicha Vienesa actúa como agente aglutinante.

Analizar las características bromatológicas y físicas de la salchicha elaborada con almidón de achira.

Ribera Pilatasig, J. H. (2006). Menciona qie el almidón de achira tiene alto contenido de amilasa, la cual es una proteína importante. Muestra una viscosidad muy alta en las temperaturas que se someten en la elaboración de pastas, lo cual permite manipular con mayor facilidad los geles calientes en comparación con otros almidones.

Lo que asegura la formaciòn de una pelicula con la proteina de la carne mejorando la capacidad de rentenciòn del agua formando de esta manera una emulsion estable que evita su ruptura y separación de solidos y liquidos en el embutido.

\section{Metodología}

El método de la sección describe en detalle cómo se llevó a cabo el estudio, incluyendo las definiciones conceptuales y operacionales de las variables utilizadas en el estudio, diferentes tipos de estudios se basan en diferentes metodologías; Sin embargo, una descripción completa de los métodos utilizados permite al lector a evaluar la idoneidad de los métodos y la fiabilidad y la validez de sus resultados, sino que también permite a los investigadores con experiencia para replicar el estudio, si su manuscrito es una actualización de un curso o anterior el estudio y el método ha sido publicado en detalle en otra parte, es posible que remitir al lector a esa fuente y simplemente dar una breve sinopsis del método de esta sección.

La presente investigación se realizó en el Centro de Producción de Cárnicos de la Facultad de Ciencias Pecuarias de la Escuela Superior Politécnica de Chimborazo. La investigación se realizó bajo un diseño experimental completamente al azar. Es decir descriptiva e inferencial. Se comparó un tratamiento testigo y tres de experimentación 5, 10, 15\% de Almidón de achira, los cuales ayudaron al desarrollo y análisis de los parámetros de estudio. Y se aplicaron

El método estadístico aplicado fue un diseño completamente al azar (DCA), debido a que las muestras a analizarse serán homogéneas para la investigación.

Los datos fueron procesados de acuerdo a los siguientes análisis estadísticos

Análisis de varianza (ADEVA) para las diferencias en las variables del análisis de la proteina y separacion de medias mediante Duncan al nivel se Significancia $\mathrm{P} \leq 0.05$ y $\mathrm{P} \leq 0.01$ 
A continuación se detalla el esquema de experimento aplicado en la presente investigación, se trabajó con 1 tratamiento testigo y 3 tratamientos relacionados con los niveles de almidón de Achira, se realizaron 3 repeticiones por cada tratamiento en 2 ensayos consecutivo, con un tamaño de unidad experimental de $2 \mathrm{Kg}$.

$\begin{array}{lcccc}\text { Tratamientos } & \text { Código } & \text { Repeticiones TUE* } & \text { Kg/Tratamiento } \\ \text { Sin aglutinantes } & \text { T0 } & 3 & 2 & 6 \\ \text { Almidón de achira 5\% } & \text { T1 } & 3 & 2 & 6 \\ \text { Almidón de achira 10\% } & \text { T2 } & 3 & 2 & 6 \\ \text { Almidón de achira 15\% } & \text { T3 } & 3 & 2 & 6 \\ \text { Total Kg } & 24 & & & \end{array}$

Modelo Estadístico

yi.j. $=\mu+\alpha \mathrm{i}+\epsilon \mathrm{ij}$

Donde:

yij: Valor estimado de la variable

$\mu$ : Media General

ai: Efecto de las formulaciones

єij: error experimental

Esquema del ADEVA

Fuente de variación Grados de libertad

Total

Tratamiento

Error experimental

Mediciones experimentales (Análisis proximal)

Se evaluará en la salchicha vienesa según lo sugerido por la Normativa Norma técnica ecuatoriana NTE INEN 1338.

Contenido de Proteína \%

Mediciones tecnológicas (Vida de anaquel 5, 10, 15 días).

Se evaluó en la salchicha vienesa según lo sugerido por la Normativa Norma técnica ecuatoriana 
NTE INEN 783:96 (Flores, I. 2001).

Perdida por goteo

pH y Acidez

\section{Metodología para la determinación de la proteína}

Pesar $1 \mathrm{~g}$ de muestra en el papel filtro, envolver e introducir en el balón de Kjendahl.

Añadir una cuchara al ras de la mezcla catalizador- elevador de la temperatura adicionar $25 \mathrm{ml}$ de ácido sulfúrico concentrado por los bordes del balón con sumo cuidado

Colocar el balón de Kjendahl en la hornilla eléctrica para su ataque durante una hora y media aproximadamente. La finalización del ataque se observa por la aparición de una solución verde esmeralda limpia. Durante la hora y media de digestión, el balón de Kjendahl se va rotando periódicamente con la finalidad de que la combustión de la materia orgánica en la muestra sea homogénea.

Dejar enfriar el producto así obtenido y adicione aproximadamente $500 \mathrm{ml}$ de agua.

Antes de iniciar el proceso de destilación, en un vaso erlemeyer añada 50ml de ácido bórico y $3 \mathrm{a}$ 4 gotas del indicador rojo de metilo. Coloque el vaso erlemeyer en el terminal del equipo de destilación de modo que el terminal quede inmerso en la solución bórica.

En el balón de Kjendahl, después de adicionar los $500 \mathrm{ml}$ de agua, añada unas cuantas granallas de zinc e inmediatamente $50 \mathrm{ml}$ de solución de soda al $50 \%$ y coloque en el equipo de destilación, ajustando bien la parte inicial de este al balón Kjendahl.

Iniciar la destilación, hasta obtener un volumen aproximado de $250 \mathrm{ml}$ de destilado en el vaso erlemeyer e interrumpa el proceso de destilación.

Titule el contenido del vaso erlemeyer con $\mathrm{HCl} 0.1 \mathrm{~N}$ hasta lograr el cambio de color en este caso de amarillo a rojo. Anote el volumen gastado.

\section{Cálculos}

$\%$ Proteína $=(\mathrm{V} \times \mathrm{N} \times 14$ x f $) /(1000$ x W $) \times 100 \%$

Donde

$\mathrm{V}=$ Volumen de $\mathrm{HCl}$ utilizado en la titulación

$\mathrm{N}=$ Normalidad del $\mathrm{HCl}$

14 = Equivalente-gramo del nitrógeno

$\mathrm{W}=$ peso de la muestra

$\mathrm{F}=$ Factor proteico

\subsubsection{Análisis Físicos (Flores, I. 2001)}


Para la determinación del pH se siguió el siguiente procedimiento

Pesar $10 \mathrm{~g}$ de muestra y añadir $100 \mathrm{ml}$ de agua destilada.

Moler la mezcla en una licuadora por un minuto.

Mezclar hasta homogeneizar.

Filtrar el contenido utilizando manto de cielo o papel filtro.

Lavar los electrodos con agua destilada.

Estandarizar el peachimetro utilizando la solución buffer 6 .

Introducir el peachimetro en el vaso de precipitación que contiene la mezcla.

Sacar el peachimetro del recipiente y "Leer"

Para la determinación de la acidez, se siguió el siguiente procedimiento

Pesar $10 \mathrm{~g}$ de salchicha.

Colocarla en un vaso de licuadora.

Añadir $200 \mathrm{ml}$ de agua destilada.

Filtrar la mezcla utilizando un manto de cielo o papel filtro.

Colocar el filtrado en un matraz de $250 \mathrm{ml}$ y aforar con agua destilada.

Tomar $25 \mathrm{ml}$ de esta solución y colocarla en otro matraz de $150 \mathrm{ml}$ de capacidad.

Añadir $75 \mathrm{ml}$ de agua destilada.

Titular con solución $\mathrm{NaOH} 0.01 \mathrm{~N}$ usando fenolftaleína como indicador.

Realizar los cálculos respectivos.

Para la determinación de la perdida por goteo se siguió el siguiente procedimiento

Pesar 500g de salchicha y empacar al vacío

Conservar en refrigeración a $5^{\circ} \mathrm{C}$.

Tiempo 5, 10 y 15 días

Pesar y registrar los valores reportados

Realizar los cálculos respectivos

\section{Resultados}

Composición nutritiva y de las salchichas vienesas elaboradas con el 5, 10, 15\% de almidón de 
achira en comparación con el tratamiento testigo.

Cuadro $\mathbf{N}^{\mathbf{0}} 1$ Reporte del analisis proximal de la salchicha vienesa

Niveles de almidón de achira

\section{Media}

\begin{tabular}{|c|c|c|c|c|c|c|c|c|c|c|}
\hline Parámetro & $\mathrm{T}_{0}$ & $\mathrm{~T}_{1} 5 \%$ & & $\mathrm{~T}_{2} 10 \%$ & & $\mathrm{~T}_{3}$ & & general & Probabilidad & $\mathrm{CV} \%$ \\
\hline Humedad \% & 69.59 & a 69.62 & $\mathrm{a}$ & 69.53 & $\mathrm{a}$ & 69.99 & a & 69.89 & 1.00 & 5.20 \\
\hline Proteína \% & 12.72 & a 12.99 & $\mathrm{a}$ & 12.91 & $\mathrm{a}$ & 12.04 & a & 12.66 & 0.80 & 11.76 \\
\hline Grasa \% & 11.11 & a 11.27 & $\mathrm{a}$ & 11.06 & $\mathrm{a}$ & 11.06 & $a$ & 11.12 & 1.00 & 15.87 \\
\hline Ceniza \% & 3.04 & a 3.06 & $\mathrm{a}$ & 2.97 & $\mathrm{a}$ & 3.27 & $c^{c}$ & 3.09 & 0.05 & 4.43 \\
\hline
\end{tabular}

Guzmán, F. 2015.

Los contenidos de proteína de las salchichas vienesas elaboradas con diferentes niveles de almidón de achira empleado como aglutinante, y el tratamiento control, no registran diferencias estadísticas $(\mathrm{P}>0.05)$, por cuanto los valores encontrados fueron de $\mathrm{T}_{0} 12.72 \% . \mathrm{T}_{1} 12.99 \%, \quad \mathrm{~T}_{2}$ $12.91 \%$, y $\mathrm{T}_{3} 12,04 \%$, estableciéndose una media general de $12.66 \%$ (Cuadro $\mathrm{N}^{\circ} 1$ ), por lo que puede indicarse que el almidón de achira utilizado como agente aglutinante no afecta el contenido proteico de la salchicha.

Los valores determinados están dentro de las exigencias requeridas por el INEN (2010), en su Norma NTE INEN 1338 , donde se indica que la salchicha escaldada debe contener como mínimo un $12 \%$, el presente trabajo supera el $12 \%$ de proteína; resultados que guardan relación con los estudios de Moreno, G. (2001) y Martínez, N. (2004), que reportan contenidos de proteína de 15.43 y $14.06 \%$ en las salchichas elaboradas a base de carne de bovino y cerdo (salchicha vienesa), mientras Salinas, M. (2010) y Capúz, N. (2014), reportaron valores de 34.84 y 11.3\%, cuando utilizaron harina de quinua y harina de amaranto en la formulación de la salchicha escaldada tipo viena, por lo tanto la salchicha vienesa estudiada presenta un aporte proteico similar que las salchichas de los estudios citados. 
Los valores de humedad en las salchichas vienesas no presentaron diferencias estadísticas $(\mathrm{P}>0.05)$, en la utilización de los diferentes niveles de almidón de achira empleado como aglutinante, así como en el tratamiento control. El contenido de humedad en las salchichas fluctuó entre 69.59 y $69.99 \%$, en el testigo y los tres tratamientos en estudio, estableciéndose una media general de $69.68 \%$ (Cuadro $\mathrm{N}^{\circ} 1$ ), lo que permite deducir que al adicionar el almidón de achira, el contenido de humedad del embutido no se altera.

Los resultados obtenidos en el presente trabajo en la elaboración de salchichas vienesas, guardan relación con los estudios de Moreno, G. (2001) Martínez, N. (2004), quienes registraron contenidos de humedad entre 64 y $66 \%$, cuando utilizaron almidones en reemplazo de la carne de res; Salinas, M. (2010) y Capúz, N. (2014), reportaron contenidos de 62.29 y $62.3 \%$, cuando utilizaron harina de quinua y harina de amaranto en la formulación de la salchicha escaldada tipo viena, los productos utilizados por estos investigadores tienen la capacidad de absorber y mantener el agua, además los valores señalados se encuentran dentro de los requisitos exigidos por el INEN (2012), en la Norma NTE INEN 1 338, donde se indica que la salchicha escaldada o cocida debe contener hasta un $65 \%$ de humedad (pérdida por calentamiento).

De acuerdo con los resultados obtenidos el contenido de grasa de las salchichas vienesas no presentaron diferencias estadísticas $(\mathrm{P}>0.05)$, en la utilización de los diferentes niveles de almidón de y el tratamiento control, reportándose contenidos entre T0 11.11 y T3 $11.06 \%$, estableciéndose un valor promedio de $11.12 \%$ (Cuadro $\mathrm{N}^{\circ} 1$ ), valores que son óptimos para la alimentación, lo que se contrapone con lo señalado en http://www.braedt.com (2010), donde se reporta que las salchichas vienesas son alimentos grasos y de un considerable contenido de sodio (sal), por lo que su consumo debe restringirse en caso de sobrepeso u obesidad; diferencia que puede deberse posiblemente a las formulaciones empleadas; Estudios nacionales en salchichas escaldadas, entre los que se mencionan a Moreno, G. (2001) y Martínez, N. (2004), determinaron contenidos de grasa entre 16.1 y $18.8 \%$; yalinas, M. (2010) y Capúz, N. (2014), 13.94\% y $9.6 \%$, al utilizar harina de quinua y harina de amaranto respectivamente.

Los resultados del presente estudio muestran valores por debajo del límite máximo permitido por INEN (2012), en la Norma NTE INEN 1338 , donde se indica que el contenido máximo de grasa total para productos escaldados debe ser de $25 \%$. Sin embargo en la actualidad se ha determinado la necesidad de bajar el contenido de grasa animal en este tipo de productos para evitar problemas cardiovasculares en los consumidores. 
Comportamiento del $\mathrm{pH}$ a los 5 días de elaboración de las salchichas Vienesas elaboradas con almidón de Achira.

Cuadro No 2 Reporte físico-química de las salchichas vienesas

\begin{tabular}{|c|c|c|c|c|c|c|c|c|c|c|c|}
\hline \multirow[b]{2}{*}{ Parámetro } & \multicolumn{11}{|c|}{ Niveles de almidón de achira } \\
\hline & $\mathrm{T}_{0}$ & & $\mathrm{~T}_{1} 5 \%$ & & $\mathrm{~T}_{2} 10 \%$ & & $\mathrm{~T}_{3} 15 \%$ & & $\begin{array}{l}\text { Media } \\
\text { general }\end{array}$ & Probabilidad & $\mathrm{CV} \%$ \\
\hline pH 5 días & 6.26 & $\mathrm{a}$ & 6.26 & $\mathrm{a}$ & 6.23 & 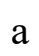 & 6.23 & $\mathrm{a}$ & 6.24 & 0.99 & 2.41 \\
\hline ph10 días & 6.15 & $\mathrm{a}$ & 6.18 & $\mathrm{a}$ & 6.22 & $\mathrm{a}$ & 6.19 & $\mathrm{a}$ & 6.18 & 0.80 & 1.72 \\
\hline ph15 días & 6.03 & $\mathrm{a}$ & 6.08 & $\mathrm{a}$ & 6.01 & $\mathrm{a}$ & 6.05 & $\mathrm{a}$ & 6.04 & 0.58 & 1.24 \\
\hline Acidez 5 días & 6.48 & $\mathrm{a}$ & 6.25 & a & 6.25 & $\mathrm{a}$ & 6.25 & $\mathrm{a}$ & 6.31 & 0.91 & 8.39 \\
\hline Acidez 10 días & 6.05 & $\mathrm{a}$ & 6.25 & $\mathrm{a}$ & 6.25 & a & 6.00 & $\mathrm{a}$ & 6.14 & 0.93 & 11.06 \\
\hline Acidez 15 días & 5.73 & $\mathrm{a}$ & 6.23 & $\mathrm{a}$ & 6.00 & $\mathrm{a}$ & 6.00 & $\mathrm{a}$ & 5.99 & 0.31 & 5.93 \\
\hline
\end{tabular}

Guzmán, F. 2015.

Los valores del $\mathrm{pH}$ inicial de las salchichas vienesas elaboradas con la adición de almidón de achira en iferentes porcentajes y el tratamiento testigo fueron los siguientes valores medios (Cuadro $\mathrm{N}^{\circ} 2$ ), T0 6.26, T1, 6.26, T2, 6.23, y T3, 6.23. Cuyo valor promedio es 6.24. A los 5 días posteriores a la elaboración del producto.

A los 10 días posteriores de la elaboración y conservación de las salchichas a una temperatura de $5{ }^{\circ} \mathrm{C}$, se reportaron valores de $\mathrm{T} 0 \quad 6.15, \mathrm{~T} 1,6.18, \mathrm{~T} 2,6.22, \mathrm{y} \mathrm{T} 3,6.19$. Con un valor promedio es de 6.18 .

Los valores reportados luego de 15 días posteriores a la elaboración y conservación de las salchichas vienesas a $5^{\circ} \mathrm{C}$ fueron de T0 6.03, T1, 6.08, T2, 6.01, y T3, 6.05. Determinándose un valor promedio es de 6.04 .

Entre los valores de $\mathrm{pH}$ reportados desde el día 5 hasta el día 15 posterior a la elaboración de la salchicha vienesa, no difieren estadísticamente $(\mathrm{P}>0.05)$, lo que nos indica que la adición del almidón de achira en la fórmula para la elaboración de la salchicha vienesa no tiene una influencia directa sobre la fluctuación en el tiempo del $\mathrm{pH}$. 
Además de acuerdo a los valores promedio a los 5 días $\mathrm{pH}$ 6.24, a los 10 días $\mathrm{pH} 6.18$ y a los 15 días pH 6.04, la acidez implícita en los valores reportados hace que la salchicha vienesa sea un producto óptimo para el consumo. Puesto que si comparamos con lo exigido por la norma técnica Ecuatoriana INEN (2010), en la Norma NTE INEN 1338, la cual menciona que el pH de los productos embutidos y escaldados es de 6.2. Se comprueba que los valores reportados están dentro de este rango.

Los valores de Acidez inicial de las salchichas vienesas elaboradas con la adición de almidón de achira en diferentes porcentajes y el tratamiento testigo fueron los siguientes valores medios (Cuadro $\mathrm{N}^{\circ}$ ), $\mathrm{T}_{0} \quad 6.48, \mathrm{~T}_{1}, 6.5 .25, \mathrm{~T}_{2}, 6.25, \mathrm{y} \mathrm{T}_{3}, 6.25$. Cuyo valor promedio es $6.31^{\circ} \mathrm{D}$. A los 5 días posteriores a la elaboración del producto.

A los 10 días posteriores de la elaboración y conservación de las salchichas a una temperatura de $5^{\circ} \mathrm{C}$, reportaron valores de $\mathrm{T}_{0} 6.05, \mathrm{~T} 1,6.15, \mathrm{~T}_{2}, 6.25, \mathrm{y} \mathrm{T}_{3}, 6.00$. Con un valor promedio es de $6.14^{\circ} \mathrm{D}$.

Los valores reportados luego de 15 días posteriores a la elaboración y conservación de las salchichas vienesas a $5^{\circ} \mathrm{C}$ fueron de $\mathrm{T}_{0} 5.73, \mathrm{~T}_{1}, 6.23, \mathrm{~T}_{2}, 6.00$, y $\mathrm{T}_{3}, 6.00$. Determinándose un valor promedio es de $5.99^{\circ} \mathrm{D}$.

Entre los valores de Acidez reportados desde el día 5 hasta el día 15 posterior a la elaboración de la salchicha vienesa, no difieren estadísticamente $(\mathrm{P}>0,05)$, lo que nos indica que la adición del almidón de achira en la fórmula para la elaboración de la salchicha vienesa no tiene una influencia directa sobre la fluctuación en el tiempo en Acidez.

Además de acuerdo a los valores promedio a los 5 días, la acidez de $6.31^{\circ} \mathrm{D}$, a los 10 días Acidez de $6.14^{\circ} \mathrm{D}$ y a los 15 días Acidez $5.99{ }^{\circ} \mathrm{C}$, estos demuestran valores ácidos. Teniendo una relación directa a con los valores de $\mathrm{pH}$ anteriormente reportados.

Los valores de Acidez reportados se encuentran dentro de lo exigido por la norma técnica Ecuatoriana INEN (2010), en la Norma NTE INEN 1338 la cual menciona que la Acidez de los productos embutidos y escaldados es de 6,8. Se comprueba que los valores reportados están dentro del rango permisible.

Pedida por goteo de las salchichas vienesas elaboradas con el 5, 10,15\% de almidón de achira en comparación con el tratamiento testigo. 
Cuadro $N^{0} 3$ Reporte perdida por goteo en las salchichas en el tiempo

\begin{tabular}{|c|c|c|c|c|c|c|c|c|c|c|}
\hline \multirow[b]{2}{*}{ Parámetro } & \multicolumn{10}{|c|}{ Niveles de almidón de achira } \\
\hline & $\mathrm{T}_{0}$ & & $\mathrm{~T}_{1} 5 \%$ & & $\mathrm{~T}_{2} 10 \%$ & & $\mathrm{~T}_{3} 15 \%$ & & Probabilidad & $\mathrm{CV} \%$ \\
\hline Peso inicial & 500.00 & $\mathrm{a}$ & 500.00 & $\mathrm{a}$ & 500.00 & a & 500.00 & $\mathrm{a}$ & 1.00 & 0.00 \\
\hline Peso 5 días & 495.00 & $\mathrm{~b}$ & 499.00 & $\mathrm{a}$ & 500.00 & & 499.25 & $\mathrm{a}$ & 0.00 & 0.25 \\
\hline Peso 10 días & 488.50 & $\mathrm{~b}$ & 498.75 & $\mathrm{a}$ & 500.00 & $\mathrm{a}$ & 497.63 & $\mathrm{a}$ & 0.00 & 0.46 \\
\hline Peso 15 días & 481.50 & $\mathrm{~b}$ & 496.25 & $\mathrm{a}$ & 500.00 & $\mathrm{a}$ & 495.00 & $\mathrm{a}$ & 0.00 & 0.88 \\
\hline Perdida por goteo inicial & 0.00 & $\mathrm{a}$ & 0.00 & $\mathrm{a}$ & 0.00 & $\mathrm{a}$ & 0.00 & $\mathrm{a}$ & 1.00 & 0.00 \\
\hline Perdida por goteo 5 días & 5.00 & $\mathrm{a}$ & 1.00 & $\mathrm{a}$ & 0,00 & $d$ & 0.75 & $\mathrm{c}$ & 0.00 & 0.63 \\
\hline Perdida por goteo 10 días & 11.50 & $\mathrm{a}$ & 1.25 & $\mathrm{a}$ & 0,00 & $\mathrm{c}$ & 2.38 & $\mathrm{~b}$ & 0.00 & 1.14 \\
\hline Perdida por goteo 15 días & 18.50 & $\mathrm{a}$ & 3.75 & $\mathrm{a}$ & 0,00 & $\mathrm{c}$ & 5.00 & $\mathrm{~b}$ & 0.00 & 2.17 \\
\hline
\end{tabular}

Guzmán, F. 2015

Según los resultados registrados en el (Cuadro $\mathrm{N}^{\circ} 3$ ), el estudio inicio con un peso de 500g para cada uno de los tratamientos $\left(\mathrm{T}_{0}, \mathrm{~T}_{1}, \mathrm{~T}_{2}, \mathrm{~T}_{3}\right)$.

Presento después de 5 días de elaboración un valor de $\mathrm{T}_{0}, 495.00 \mathrm{~g}$; luego de 10 días de conservación fue de $488.50 \mathrm{~g}$; a los 15 días posteriores de conservación se registró un valor de 481.50g; La perdida por goteo de acuerdo a los resultados reportados fue de $18,50 \mathrm{~g}$ en el periodo de tiempo en el que se realizó el estudio.

Con el $\mathrm{T}_{1} 5 \%$ de almidón de achira se registró un peso de 499g, luego de 5 días de elaboración (Cuadro No8); a los 10 días el peso fue $498,75 \mathrm{~g}$; y a los 15 días el valor fue de 496,25 g. Determinándose una perdida por goteo de $3,75 \mathrm{~g}$ al cabo de 15 días de estudio de las salchichas. Esto se debe a que el porcentaje de almidón de achira añadido a la fórmula para la elaboración de la salchicha no encapsula toda el agua presente en el producto. $\mathrm{Al}$ adicionar $\mathrm{T}_{2}$ un $10 \%$ de almidón de achira en la elaboración de salchicha vienesa se puede observar que no existe una fluctuación del peso en el tiempo puesto el peso inicial de $500 \mathrm{~g}$, se mantiene hasta el final del tiempo de estudio es decir no existe perdida por goteo, esto se debe a que el porcentaje de almidón de achira incluido en la fórmula encapsula en mayor grado el agua presente en el producto.

El peso de las salchichas elaboradas con adición del $T_{3} 15 \%$ de almidón de achira fluctuó en el tiempo (cuadro), a los 5 días 499,25 g; a los 10 días 497,63 g; y a los 15 días un valor de 495,00 g. 
Determinándose una perdida por goteo de $5,00 \mathrm{~g}$ al cabo de 15 días de estudio. Esto se debe a que el almidón de achira añadido a la fórmula ha encapsulado el agua hasta sobresaturarse y empieza un proceso de retrogradación, lo que hace que el agua encapsulada se libere y salga del producto arrastrando algunas sustancias sólidas.

\section{Conclusion}

- El empleo de los diferentes niveles de almidón de achira como agente aglutinte en la elaboración de la salchicha vienesa, no presentó diferencias estadísticas en la composición nutritiva, y se pudo determinar valores que están dentro de los rangos exigidos por el INEN (2010), en su Norma NTE INEN 1338.

- La Salchicha Vienesa presenta valores promedio de, $12.66 \%$ de proteína, superiores a los exigidos por la norma INEN 1338.

- En propiedades físicas la salchicha vienesa como el pH y la acidez no presentó una diferencia estadística significativa entre las medias reportadas para tos los tratamientos de estudio, cuyos valores fueron a los 5 días pH 6,24; a los 10 días 6,18; a los 15 días 6,04 y la Acidez a los 5 días $6,31^{\circ} \mathrm{D}$; a los 10 días $6,14^{\circ} \mathrm{D}$ y a los 15 días $5,99^{\circ} \mathrm{D}$, tanto el pH como la acidez fluctuaron en valores considerados ácidos, siempre manteniéndose durante el periodo de tiempo de estudio, en el rango permisible para este tipo de productos, exigidos en la INEN (2010), en su Norma NTE INEN 1338.

- Con la utilización del almidón de achira en la formulación de la salchicha vienesa se pudo comprobar que luego de 15 días de conservación no se observó una reducción en el peso de las salchichas es decir no se presentó perdida por goteo marcado, debido a la capacidad aglutinante y gelificante del almidón de Achira.

- Al utilizar el $10 \%$ de almidón de achira en la elaboración de la salchicha vienesa obtuvo los mejores valores durante el análisis de proteína, propiedades físicas y la vida de anaquel.

- discutido.

\section{Agradecimiento}

Un agradecimiento a Escuela Superior Politecnica de Chimborazo por su apoyo al desarollo de esta investigaciòn mediante la facilidad para el uso de sus laboratorios de Bromatologia y Nutriciòn, laboratorio de microbiologia y parasitologia, Unidad de procesamiento de alimentos y planta de procesamiento de carnicos.

A la ingeniera Gabriela Bayas tecnico de la planta de procesamiento de alimentos y procesmiento de carnicos por la guia y apoyo durante el proceso de elaboraciòn del 
producto en estudio.

A la ingeniera Sandra Lopez docente de la Carrera de industrias Pecuarias quien con sus conocimientos guió el desarollo de los analisis proximal y fisico quimico del producto salchicha vienesa.

Al PhD Ivan Flores director de la Carrera de Agroindiudtrias e Industrias Pecuarias, director del laboratorio de microbilogia y parasitologia quien participo activamente como guía durante el desarrollo de los estudios microbiológicos del producto en estudio.

\section{Referencias Bibliograficas}

American Psychological Association. (1972). Ethical standards of psychologists. Washington, DC: American Psychological Association.

Alcocer, M., \& Enrique, L. (2011). Utilización de Fibra de Trigo en la Elaboración de Salchichas Dietéticas Tipo Vienesa(Bachelor's thesis).

Asqui, P., \& Hernan, M. (2011). Calidad Nutritiva, Microbiológica y Organoleptica de la Salchicha Tipo Vienesa con Diferentes Niveles de Inclusión de Harina de Arveja (Bachelor's thesis).

Aktaş, N., \& Genccelep, H. (2006). Effect of starch type and its modifications on physicochemical properties of bologna-type sausage produced with sheep tail fat. Meat Science, 74(2), 404408.

Bonete, M., Urquizo, C., Guevara, R., \& Yánez, P. (2016). Estudio de cuatro tubérculos y raíces tuberosas no tradicionales de la sierra centro de Ecuador y su potencial de uso en platos de autor. Qualitas, 12, 37-67.

Borja, M., \& Rolando, N. (2011). Evaluación de cuatro Niveles (1.25, 2.5, 3.75 y 5.0) de fFécula de Maíz en la Elavoración de Salchicha Vienesa (Bachelor's thesis).

CARBALlO, B. (2000). Manual de bioquímica y tecnología de la carne. Madrid: Ediciones A.

Capúz, N. 2014 Sustitución parcial de haría de trigo por harina de amaranto variedad INIAP alegría (Amaranthus caudatus) y sus incidencia en las características físico-químicas y sensoriales de salchicha escaldada. Tesis de grado Universidad Técnica de Ambato. Facultad de Ciencias e Ingeniería de alimentos.

Diestre, A. 1992. Principales problemas de calidad de la carne en el porcino. Alimentación. Equipos y Tecnología.

Ecuador, Instituto nacional ecuatoriano de normalización (INEN). 2012. Norma NTE INEN1 1338:12. Quito, Ecuador.

Ecuador, Instituto nacional ecuatoriano de normalización (INEN). 2012. Norma NTE INEN1 1529-8. Quito, Ecuador. 
Ecuador, Instituto nacional ecuatoriano de normalización (INEN). 2012. Norma NTE INEN1 1529- 5: Quito, Ecuador.

Ecuador, Instituto nacional ecuatoriano de normalización (INEN). 1996. Norma NTE INEN 783 Quito, Ecuador.

Essien, E. 2005. Fabricación de embutidos: principios y práctica. Acribia.

Forrest, J.C., Aberle, E.D., Hedrick, H.B., Judge, M.D. and Merkel, R.A. 1979. Fundamentos de Ciencia de la Carne. Editorial Acribia (España). 364 p. Guerrero, L.

Franco, J., Feed, O., Bianchi, G., Garibotto, G., Ballesteros, F., Nan, F., \& Bentancur, O. 2008. Parámetros de calidad de carne en cinco músculos de novillos Holando durante la maduración post-mortem I. Calidad instrumental. Agrociencia, 12(1), 61-68.

García-García, E., \& Totosaus, A. 2008. Low-fat sodium-reduced sausages: Effect of the interaction between locust bean gum, potato starch and $\kappa$-carrageenan by a mixture design approach. Meat Science, 78(4), 406-413.

García, S. H., \& Vera, N. G. 2010. Efecto de la Adición de Harina de Cáscara de Naranja sobre las Propiedades Fisicoquímicas, Textuales y Sensoriales de Salchichas Cocidas. Nacameh, 4(1), 23-36.

Girard J. 2010. Tecnología de la carne y de los productos cárnicos Zaragoza-España. Edit. Acribia. Pp. 139-144.

Grepe, N. 2001. Elaboración de productos cárnicos. $1^{\text {a }}$ ed. México, México. Edit. Iberoamérica. Pp 24-26.

Gutierrez, D. C., Ruiz, B. S., \& Peñafiel, C. E. (2002, January). Utilización de harina texturizada y concentrado funcional de soya, carragenina y piel de cerdo en salchichas tipo huacho con bajo tenor graso. In anales científicos (p. 197).

http://www.accefyn.org.co, 2012.

http://www.braedt.com 2010. Salchichas.

http://www.ecured.cu/achira-saju 2013.

http: //www.funtha.gov.ve/docalmidon-saju, 2007.

http://www.ucsg.edu.ec/catolica/secundarias/html/facultad_medicina. 2010.

http://izarzugaza.com/index.php?option=com_content\&view=article\&id=139:tipos-decarne\&catid=15: wiik\&Itemid=107\&lang=es. 2013.

Hui, Y. H., \& Legarreta, I. G. 2006. Ciencia y tecnología de carnes. Limusa.

Lawrie, H. 2002 Ciencia de la carne. $2^{\text {a }}$ ed. Zaragoza, España. Edit. Acribia. pp 10-25.

Luna J. 2010. Manual de Proceso del Rastro y Planta Industrializadora de Productos Cárnicos de la Posta Zootécnica Michoacán México.

López, G Y Carballo, B. 1991, Tecnología de la carne y de los subproductos cárnicos $1^{\mathrm{a}}$ ed. Edit. 
AMV Ediciones. Madrid-España pp. 54, 55.

Morales, R. 2001. Características físicas, químicas y organolépticas del almidón de "achira". Revista de la Academia Colombiana de Ciencias exactas, físicas y naturales, 13(51), 357368.

Mejía, L. F. 2003. Practica de almidones, gelatinización y retrogradación. Universidad Nacional de Colombia sede Palmira. P, 4.

Moreno, G. 2001. Utilización de fécula de papa en la elaboración de salchicha vienesa. Tesis de Grado. Facultad de Ciencias Pecuarias, ESPOCH. Riobamba, Ecuador. pp 38-57.

Meyer, M. y Paltrinieri, G. 1996, Elaboración de Productos Cárnicos 1 ${ }^{\mathrm{a}} \quad$ ed. Edit. Trillas S.A. México-México, pp. 21, 22, 23, 25.

Mira, J. 1998. Compendio de tecnología y ciencia de la carne. $1^{\text {a }}$ ed. Ed. AASI. Riobamba, Ecuador. pp. $10,32$.

Martínez, N. 2004. Evaluación de cuatro niveles $(1.25,2.5,3.75$ y 5.0\%) de fécula de maíz en la elaboración de salchicha vienesa. Tesis de Grado. Facultad de Ciencias Pecuarias, ESPOCH. Riobamba, Ecuador. pp 35-57.

Ochoa lucero, J. F. 2015. Elaboración de salchicha Frankfurt con la utilización de harina de colacasia esculenta (Malanga Blanca).

Obispo, N. E., Pares, P., Hidalgo, C., Palma, J., \& GODOY, S. 2001. Consumo de forraje y ganancia diaria de peso en bovinos de carne en crecimiento suplementados con fuentes proteicas (Forage intake and average daily gain of young heifers supplemented with protein sources).

Pamplona J. 2006. Enciclopedia De Las Plantas Medicinales. Buenos.

Picallo, A. 2002. El análisis sensorial como herramienta de calidad de carne y productos cárnicos de cerdo. Buenos Aires Argentina. Edit. INTA.

Pietrasik, Z. 1999. Effect of content of protein, fat and modified starch on binding textural characteristics, and colour of comminuted scalded sausages. Meat Science, 51(1), 17-25.

Price, J. F., \& Schweigert, B. S. (EDS.). 1994. Ciencia de la carne y de los productos cárnicos (pp. 125-129). Acribia.

Rodríguez Caeiro, M. Elaborador de productos cárnicos: manual operativo de elaboración y preparación de masas, piezas y productos cárnicos/Ma. José Rodríguez Caeiro (No. 664.902 R63 2005.).

Rebollo, M. R. 2000. Utilización de aditivos. Cárnica 2000, 27(201), 65-80.

Ríos, K. C. 2011. Tecnología de alimentos. Ediciones de la U. Bogotá.

Rojas, A. T. 2003. Guía de procesos para la elaboración de productos cárnicos. Siglo Del Hombre Editores SA.

Saetama, L., \& Beatriz, M. 2015. Evaluación de la carne de llama y alpaca en sustitución parcial y 
total de la carne de bovino y cerdo en la obtención de mortadela.

Salinas, M. 2010 Efecto de la sustitución de harina de Trigo por harina de quinua (Chenopodium quinoa) para la formulación y elaboración de salchichas tipo vienesa con características funcionales. Tesis de grado Universidad Técnica de Ambato. Facultad de Ciencias de alimentos.

Salinas Rueda, M. E. (2010). Efecto de la sustitución de harina de trigo por harina de quinua (chenopodium quinoa) para la formulación y elaboración de salchichas tipo vienesa con características funcionales.

Santacruz, S. (1995). Estudio de las características del almidón de achira (Canna edulis).

Srivastava, J., \& Vankar, P. S. 2010. Canna indica flower: New source of anthocyanins. Plant Physiology and Biochemistry, 48(12), 1015-1019.

Vera, P., \& Rocío, D. 2006. El estudio de la adición de almidón modificado en la elaboración de Salchicha

World Health Organization, \& Codex Alimentarius Commission. (2005). Codex Alimentarius. FAO/WHO. Código de prácticas de higiene para la carne CAC/RCP 58/2005. 


\section{PARA CITAR EL ARTÍCULO INDEXADO.}

Guzmán Acán, F., Bonifaz, V. de A., \& Vayas Castillo, G. (2019). Evaluación Del Efecto Aglutinante Del Almidón De Canna Indica L. (Achira), En La Elaboración De la Salchicha Vienesa. Ciencia Digital, 3(2.6), 388405. https://doi.org/10.33262/cienciadigital.v3i2.6.573

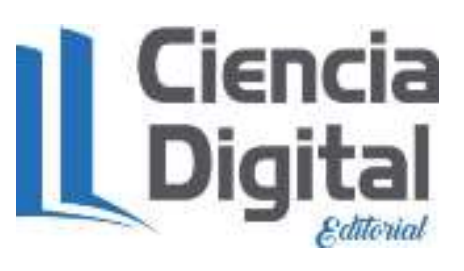

El artículo que se publica es de exclusiva responsabilidad de los autores y no necesariamente reflejan el pensamiento de la Revista Ciencia Digital.

El artículo queda en propiedad de la revista y, por tanto, su publicación parcial y/o total en otro medio tiene que ser autorizado por el director de la Revista Ciencia Digital.
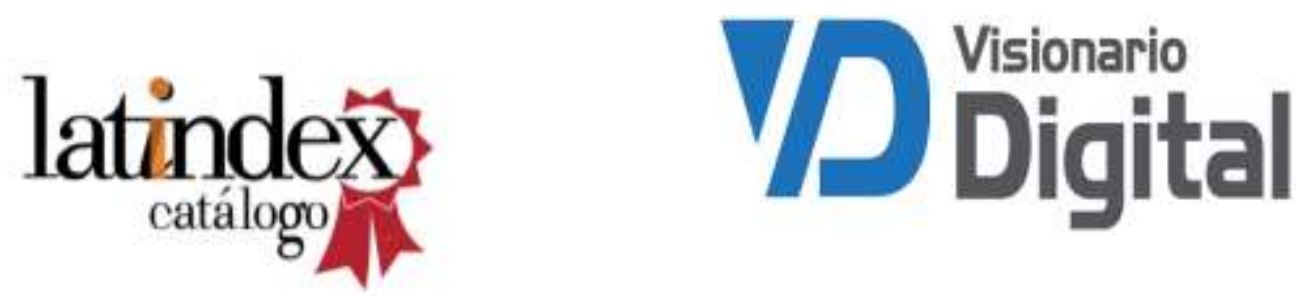